extend our fundamental knowledge of the processes so as to broaden the basis of possible applications. In general, therefore, the trend of thought is towards what may be called biological means of fertility control, involving systemic treatment, and away from local action of a mechanical or chernical nature. Systemic treatment is, of course, most easily made by mouth, in other words by 'the pill'.

Biological control involves one or other of three things: $(a)$ the prevention of formation or release of germ cells in male or female; $(b)$ the prevention of fertilization; (c) the prevention of implantation of the fertilized egg. The first of these involves either preventing the release of ova from the ovary or the formation of spermatozoa in the testis. Spectacular progress has been made in the past few years towards the use of orally active progestagens (the 'Pincus pill') to depress the ovary-stimulating activity of the anterior pituitary body and thus to prevent release of the ovum. The Pincus pill has been the subject of extended trials, and is likely to come into routine use to at least a limited extent. The outstanding problem of the Pincus pill is not as to whether it works -it certainly works - but as to the extent of uncomfortable side-effects and of possible hazards arising from the long-term inhibition of a vital gland and the prolonged induction of an artificial rhythm in the uterus.

Work on the inhibition of spermatogenesis by oral medication is not so far advanced. Obvious disadvantages attend the depression of ovary or testis activity by the use of substances, particularly steroid hormones, reducing pituitary gonadotrophic activity; but encouraging results on the direct inhibition of spermatogenesis without interference with the androgenic interstitial tissue have been obtained by the use of various non-steroidal compounds. In any event, there is little doubt that a pill for males will be forthcoming in the future. When this happens the obvious course will be for husband and wife to take, say, yearly shifts in consuming pills, so as to minimize the risk of adverse effects arising in either sex through indefinitely prolonged treatment.

So far, little progress has been made with the problem of preventing fertilization by biological means in circumstances where both male and female are producing viable germ cells, that is to say, in preventing effective contact between egg and sperm. It is possible, though not certain, that the most hopeful line of work here is towards immunizing the female against spermatozos and thence against pregnancy, a possibility which arises from the fact that spermatozoa are antigenic when administered parenterally to animals of a different species from the donor, and that the antibodies raised are organ- rather than species-specific. A serious difficulty, however, lies in obtaining an effective concentration of antibody at the necessary site of action within the female reproductive tract, and only recently, by the use of adjuvants, has it been possible to obtain any serious indications of a positive result. However, other immunological approaches to fertility control exist.

Work on the prevention of implantation raises the important question as to what constitutes conception. Biologically there can be little doubt that implantation of the fertilized egg in the uterus, not fertilization of the egg, constitutes conception, and that contraception can therefore properly be exercised up to the time of implantation. This is important because much interesting work is now being carried out on inactivating the fertilized egg or preventing the changes in the endometrium necessary for implantation. A method of fertility control based on such work would have the great advantage of requiring retrospective action rather than action anticipating the uncertain contingency of exposure to the risk of pregnancy. Scope for inactivating the fertilized egg is perhaps limited, but there are many possibilities of disturbing the hormonal, metabolic, pharmacological, or neurohumoral relationships necessary for the development of progestational changes in the uterus. A particularly interesting line of thought in this field arises from the observation that newly mated female mice fail to become pregnant if exposed to the smell of alien males from a different strain. The effect is exerted first through nervous and then through hormonal pathways, and the observation is evidently of great interest and potentiality; the attractive idea of a contraceptive perfume is no longer quite incredible.

In conclusion, it must be remembered that the best methods will be of little value in countries faced. with an explosive growth of population unless people can be persuaded to use them, and this may not be easy in countries conditioned to the idea of a high birth-rate and a high death-rate, and in which public health measures have reduced the death-rate so rapidly that the social background has had little chance to adjust itself. But at least we should ensure that demographic and personal aspects of human reproduction are as widely understood as possible, and that simple and reliable methods of fertility control are available for those who wish to use them. In this we have far to go. Established methods of fertility control, being archaic in principle, are a disgrace to science in this age of spectacular achievement. In general, I am in favour of science pushing on in every possible direction, but I sometimes think that the further exploration of inner man would be more immediately useful than the exploration of outer space.

\title{
SAFE HANDLING OF RADIOACTIVE SUBSTANCES
}

N 1949 a printed version of the booklet Introductory Manual on the Control of Health Hazards from Radioactive Materials, prepared by the Atomic Energy Research Establishment, was issued by the Medical Research Council. This manual has been in great demand by those working with or just beginning to work with radioactivity, but, as it has not been

* Privy Council. Medical Researeh Council Memorandum No. 39 Introductory Manual on the Control of Health Hazards from Radioactive Materials. (Committee on Protection against Ionizing Radiations.) Pp. vi +21 . (London: H.M.S.O., 1961.) 18. 9d. net. available for some years, a new edition*, commissioned by the Medical Research Council's Committee on Protection against Ionizing Radiations, is timely and welcome. It is intended to give guidance on the principles of safe working to those handling radioactive substances in laboratories and elsewhere, and contains a valuable summary of the recommendations on maximum permissible doses and the state of legislation in the United Kingdom at the end of 1960. 
In Section 3, which follows a brief introductory section and a descriptive section on the general nature of the hazards, are given the present recommendations on maximum permissible levels of radiation for those occupationally exposed to radiation and for those working nearby; these are based on the Medical Research Council's evaluation of the recom. mendations of the International Commission on Radiological Protection. In addition to listing the levels for external radiation, examples are given of actual values of maximum permissible body burdens and maximum permissible concentrations in air and water.

There is a brief outline of the measurement of radiation dose and dose-rate in Section 4; monitoring for neutrons is outside the scope of the manual. Due consideration is given to the use and value of film dosimeters, but there is no mention of whether any film-badge service is generally available. In this section reference is made to the selection of radiological safety officers to ensure that protection measures in a particular department are carried out; and to the appointment of an appropriately qualified physicist as 'radiological protection adviser' to advise the head of each department. These officers are akin respectively to the departmental radiation supervisor and university radiation protection officer recommended in the recently published Code of Practice for the Protection of Persons exposed to Ionising Radiations in University Laboratories (The Association of Universities of the British Commonwealth, London, 1961). It is also pointed out in the manual that the radiological protection adviser should work in close association with a qualified medical adviser. These officers can also be compared with the "competent persons" in the Factories Acts Regulations whose duties are to "exercise special supervision with regard to the requirements of these Regulations and to assist in enforcing the observance of them". (Factories, The
Ionising Radiations (Sealed Sources) Regulations, 1961 (London, H.M.S.O., 1961); Ministry of Labour, Factories Acts, 1937-1959, Unsealed Sources Regulations, Preliminary Draft of Regulations (London, H.M.S.O., 1961).)

Sections 5 and 6, on "Acceptable and Hazardous Amounts of Radioactive Material", contain a table classifying isotopes according to their relative radiotoxicity. This table is in line with the table in the first schedule of the Unsealed Sources Regulations. Laboratories are graded as $A, B$ or $C$ according to the quantity of radioactivity involved and its radiotoxicity; in addition, account is taken of the nature and complexity of the processes involved. This method of classification is the one that is most often given in codes of practice for safe handling of radioactive materials; however, it is not the only one, and practical experience has shown that other systems of classification of radiation and contamination give effective control of exposure to radiation.

The remaining sections of the manual cover protective clothing and devices; exhortations on 'do's' and 'don'ts' when handling radioisotopes; eating, smoking and washing; storage; a valuable section on disposal of radioactive wastes which takes account of what the situation will be when the Radioactive Substances Act, 1960, comes into force; and a final short section giving advice on where to apply for particulars concerning transport of radioisotopes.

The bibliography covers publications issued up to the end of 1960 . In the field of radiological safety so much coding and regulation is in progress that there have been at least three major documents issued since the manual was published. With this accretion of regulations and advice the manual is invaluable for all those concerned with the use of radioactive substances and the control of health hazards as a handy summary of authoritative advice at a given point in time.

\section{NEUTRON CAPTURE THERAPY}

$\mathrm{N}$ EUTRON capture therapy is an experimental radiotherapeutic procedure involving new concepts in the treatment of cancer. This treatment is a means of achieving selective irradiation of a tumour tissue by inducing throughout that tissue radioactivity of high biological efficiency. The radioactivity is induced in situ by means of the capture of thermal neutrons by a suitable target element. To develop the most efficient system, a target element is chosen which, on thermal neutron capture, decays immediately through emission of energetic heavy particles such as $\alpha$-particles. Since the range of heavy particles of $2-4-\mathrm{MeV}$. energy in tissue is very short, of the order of less than $15 \mu$, the resulting radiation effects are limited to the region of origin. Because radioactive decay is practically instantaneous and results in a stable element, physiological dispersion from the origin is prevented. Only those elements which have a high thermal-neutron capture cross-section, such as boron-10, lithium-6, and uranium-235. are useful. This suggestion was first put forward in 1936 by Locher. The utilization of thermal neutron capture by boron-10 for the treatment of human-brain tumours, chiefly glioblastoma multiforme, has been under investigation at the Medical Research Center of Brookhaven National Laboratory, New York, since 1949.
An account of the biological effectiveness of thermal neutrons and of heavy particles from the ${ }^{10} \mathrm{~B}(\mathbf{n}, \alpha)^{7} \mathrm{Li}$ reaction for the rabbit's ear and its utilization for neutron capture therapy has been given by Yaskazu Lucas Yamamoto (Yokohama Medical Bulletin, 12, No. 1, February 1961).

Throughout this series of cases, the pathological effects of thermal neutrons and of the heavy particles from the ${ }^{10} \mathrm{~B}(\mathrm{n}, \alpha)^{7} \mathrm{Li}$ reaction on human skin occurring at the point of neutron entry have been known as one of the most vexing side-effects of neutron-capture therapy. Among other side-effects such as boron toxicity, radiation parotitis, and radiation conjunctivitis, it has particularly attracted the attention of investigators, and an effort has been made to prevent or reduce the complication. In reviewing the brain tumour cases treated by neutron-capture therapy at the Medical Research Center, Yamamoto and his co-workers have encountered varying degrees of skin reactions in a large percentage of the cases ranging from erythema to severe radiation dermatitis.

In a previous series of experiments on human brain tumours, attempts were made to decrease skin radiation by decreasing the boron concentration in the skin while maintaining a maximum concentration in the neoplastic tissue in the brain. The methods 